\title{
INVESTIGACIÓN FORMATIVA EN LA ASIGNATURA DE ECOLOGÍA INDUSTRIAL
}

\author{
Salazar Zegarra, Sonia Mirtha
}

\section{Resumen}

En países en vía de desarrollo como el Perú, se considera necesario que el resultado tanto de la formación teóricapráctica como de la investigación aplicada en las Escuelas de Ingeniería Industrial, sean propuestas de mejora y crecimiento industrial en armonía con el medio ambiente, ello podrá ser alcanzado por profesionales capaces, innovadores y con valores ambientales. Para alcanzar esta meta, la Facultad de Ingeniería de la Universidad Católica Santo Toribio Mogrovejo propone, como parte del currículo, el desarrollo de la investigación formativa como eje transversal de sus asignaturas. Así este trabajo de investigación se centra en el diseño y desarrollo de la intervención en aula para la asignatura de Ecología Industrial, correspondiente al IV ciclo de Ingeniería Industrial, durante los semestres 2007- I y 2008-I, empleando como estrategia pedagógica de base la investigación formativa a fin de lograr la mejora del aprendizaje- enseñanza.

Los logros del aprendizaje se recogen con diversos instrumentos de evaluación, en particular con reportes de investigación que hacen operativos, los procesos de investigación formativa. Mediante estos reportes se evidencia que dicha estrategia, posibilita la formación de profesionales que la sociedad necesita, al crear espacios que posibilitan integrar conocimientos con la aplicación específica de los mismos, de forma que el estudiante realiza acciones de práctica, que le permitirán desarrollar competencias para la realización de investigación en sentido estricto. La generación de situaciones problemáticas iniciales de Ecología Industrial, en torno a una industria en particular, ha permitido al estudiante organizar y desarrollar conocimientos, aunque sean conocimientos ya existentes, aplicándolos en torno a sus expectativas y condiciones académicas profesionales específicas.

Los resultados alcanzados evidencian que, con un diseño modelo $T$ para la asignatura de Ecología Industrial y empleando como estrategia base la investigación formativa, se desarrollan en los estudiantes procesos en y para la investigación, incorporando progresivamente los contenidos propios de la asignatura en una aplicación industrial concreta, alcanzando con éxito los logros de aprendizaje establecidos, desarrollando además responsabilidad con el aprovechamiento industrial y cuidado de los recursos naturales. Colateralmente, este tipo de intervención en aula ha posibilitado, el desarrollo armónico de las tareas de investigación, docencia y responsabilidad ambiental del profesor universitario.

\section{ABSTRACT}

In developing countries as Peru, it is considered necessary that the result both of the theoretical - practical formation(training) and of the research applied in the Schools of Industrial Engineering, should be proposed of improvement and industrial growth in harmony by the environment, it will be able to be reached by capable, innovative professionals and with environmental values. To reach this goal, the Faculty of Engineering of the Catholic University Saint Toribio Mogrovejo proposes, as part of the curriculum, the development of the formative investigation as transverse axis of yours subjects. This way this work of research centres on the design and development of the intervention on classroom for the subject of Industrial Ecology, corresponding to the cycle IV of Industrial Engineering, during the semesters 2007-I and 2008-I, using as pedagogic strategy of base the formative investigation in order to achieve the improvement of the learning - education.

The achievements of the learning gather with diverse instruments of evaluation, especially with reports of research that they make operative, the processes of formative investigation. By means of these reports there is demonstrated that the above mentioned strategy, it makes possible the professionals' training that the society needs, on having created spaces that they make possible to integrate knowledge with the specific application of the same ones, so that the student realizes actions of practice, which will allow him to develop competitions for the accomplishment of research in strict sense. The generation of problematic initial situations of Industrial Ecology, around an industry especially, has allowed to the student to organize and to develop knowledge, though it is already existing knowledge, applying them concerning yours expectations and academic professional specific conditions.

The reached results demonstrate that, with a design I shape $T$ for the subject of Industrial Ecology and using as strategy base the formative investigation, processes develop in the students in and for the research, incorporating progressively the own contents of the subject in an industrial concrete application, reaching successfully the achievements of learning established, developing in addition responsibility with the utilization industrial and taken care of the natural resources. Colateralmente, this type of intervention in classroom has made possible, the harmonic development of the tasks of research, teaching and environmental responsibility of the university teacher.

\section{INTRODUCCIÓN}

La exigencia de la formación profesional en Ingeniería es de vital importancia para países en vías de desarrollo, como el Perú. Es una necesidad que las Escuelas de Ingeniería, en particular las de Ingeniería Industrial, formen profesionales innovadores que realicen propuestas de mejora y crecimiento industrial del país en armonía con el medio ambiente, de acuerdo a la exigencia de la formación profesional como en la investigación aplicada. Al respecto en [1] se menciona que existe una relación biunívoca entre educación y

\footnotetext{
${ }^{a}$ Escuela de Ingeniería Industrial, Universidad Católica Santo Toribio de Mogrovejo, Chiclayo, Perú.
} 
desarrollo, ya que son los sistemas educativos dominantes los que determinan el tipo de sociedad y de individuo que prevalece $\mathrm{y}$, por consiguiente, el grado, la forma y, sobre todo, la orientación del desarrollo que se pretende lograr. Tomando en cuenta este aspecto, la asignatura de Ecología Industrial se integra a la Educación para la Sostenibilidad (ONU - UNESCO), pues se abordan aspectos esenciales de crecimiento industrial relacionados con la construcción de sociedades y futuros más sostenibles.

Como parte de su forma de ser la Facultad de Ingeniería de la Universidad Católica Santo Toribio Mogrovejo, propone dentro del currículo el desarrollo de la investigación formativa como eje transversal para las asignaturas de formación profesional, unida, en el caso particular de Ecología Industrial, al concepto de sostenibilidad.

Al respecto, se coincide con [2] cuando se menciona que por la misión investigativa la universidad debe generar conocimiento descriptivo, explicativo, predictivo y tecnológico, mediante las investigaciones de grado y las de los Centros de Investigación, de esta manera se desarrolla investigación científica en sentido estricto, sin embargo por la función pedagógica la universidad debe centrar su atención en la investigación formativa. A su vez en [3] se menciona que las universidades deben reestructurar sus espacios de producción, difusión y transferencia del conocimiento, empleando la investigación como medio de cambio. Así es necesario, desarrollar procesos académicos de enseñanza y aprendizaje que propongan y desarrollen estrategias para potenciar la investigación, integrándola en el currículo como eje transversal.

La articulación de la investigación con la formación profesional tal como propone [4], se desarrolla incorporando en el currículo una metodología de desarrollo de contenidos relacionados con los métodos y problemas de investigación propios de cada disciplina de la formación profesional. También menciona que, una docencia investigativa enlazada con el currículo académico, aporta al desarrollo de la función de la investigación propia de la institución universitaria y pone al alcance de profesores y estudiantes la cultura de la investigación.

Así mismo se coincide con [5] cuando mencionan que, el uso de estrategias didácticas para el aprendizaje de las ciencias, tales como la investigación orientada con la secuenciación adecuada de contenidos, posibilita un proceso de formación en investigación análogo a la formación inicial de futuros investigadores y, por ello, el modelo de aprendizaje se denomina de investigación orientada o formativa tal como es definida por [6]. Como características resaltantes de la investigación formativa, se mencionan: a) no se orienta a la formación de conocimiento objetivamente nuevo, sino a la apropiación significativa de conocimiento ya elaborado, b) tiene una intención curricular, en el sentido de ser camino para el desarrollo de procesos de enseñanza-aprendizaje, vinculados con objetos de conocimiento predeterminado, c) se destaca como estrategia pedagógica para el desarrollo del currículo y, d) los agentes investigadores son sujetos en formación profesional.
Como técnicas de investigación formativa se señalan, los ensayos estructurados, el método ABP, los Preseminarios investigativos y las Monografías Investigativas, entre otros. Se reconoce las bondades de esta última pues, un trabajo monográfico correctamente estructurado y bien desarrollado, constituye un excelente ejercicio de investigación, en el que se integran la apropiación autónoma y significativa de contenidos académicos, el desarrollo de procesos cognitivos complejos y la adquisición de competencias significativas [8]

Así, este trabajo se centra en el diseño de intervención de aula para la asignatura de Ecología Industrial, correspondiente al III ciclo de Ingeniería Industrial en los semestres, 2007-II y 2008-I, empleando como estrategia pedagógica de base la investigación formativa. A fin que el estudiante proponga la evolución de modelos ecológicos para una industria en particular, mediante la elaboración de una monografía investigativa donde se tome en cuenta las interacciones de la industria con la biosfera, bajo un criterio de armonía con la naturaleza y evidenciando la mejora de la conciencia ambiental, mediante el diseño de sistemas ecológicos industriales y ciclos de vida de los productos.

La asignatura de Ecología Industrial de acuerdo a lo planteado por [9], desarrolla el concepto de ecología industrial como un producto de la evolución de los paradigmas sobre manejo ambiental y de la integración de nociones de sostenibilidad en los sistemas económicos y ambientales, en los cuales los procesos productivos son concebidos como parte integral del ecosistema. Este concepto surge de la percepción de que, la actividad humana está causando cambios inaceptables en los sistemas básicos de soporte ambiental. A su vez [10] destacan que, es imprescindible incorporar la educación para la sostenibilidad, como un objetivo clave en la formación de los futuros ciudadanos y ciudadanas, para hacer comprender la necesidad de realizar acciones que contribuyan a un futuro sostenible en los diferentes ámbitos: consumo responsable, actividad profesional y acción ciudadana.

Los resultados alcanzados evidencian que, la investigación formativa permite a los estudiantes desarrollar procesos en y para la investigación, incorporando progresivamente los contenidos propios de la asignatura en una aplicación industrial concreta, desarrollando además responsabilidad en el aprovechamiento y cuidado de los recursos naturales. Colateralmente, se logra el desarrollo armónico de las tareas de investigación, responsabilidad ambiental y docencia del profesor universitario.

\section{METODOLOGÍA}

Los referentes teóricos presentados, orientan el diseño de los materiales empleados en el proceso de enseñanza-aprendizaje, a saber: a) diseño del sílabo de asignatura de Ecología Industrial aprobado por Dirección de Escuela de Ingeniería Industrial, b) lecturas varias conformando 11 separatas temáticas, c) 02 casos emblemáticos, d) 02 videos y películas temáticas, e) 01 formato de reportes de visita técnicas, f) 01 ficha guía para la presentación del trabajo de investigación 
formativa, g) 01 ficha esquema para exposición final, teniendo como instrumentos de evaluación: a) pruebas de ensayo, b) reportes de visitas técnicas, c) 01 listas de cotejo para la presentación de monografía investigativa.

Los resultados de los indicadores de logro, se recogen mediante las fichas de evaluación individual y en pares, tanto del trabajo desarrollado en aula como de las tareas previstas, asistencia a asesoría, participación oral y sustentación pública del trabajo de investigación formativa.

Respecto a la metodología de trabajo para la elaboración de la monografía investigativa, en el transcurso del proceso de enseñanza-aprendizaje de Ecología Industrial y en un marco de investigación formativa, ella se inicia con la delimitación del problema de investigación que será el núcleo temático alrededor del cual se indaga continuamente, ya que está articulado con los contenidos de la asignatura establecidos. Por ello existe un continuo acercamiento y redimensionando de los contenidos de enseñanza situándolos en torno a una situación problemática formulada como, ¿Cuál es el diseño ecológico tipo III que evidencia de mejora manera los contenidos de Ecología Industrial, para una industria en particular?

A continuación, y en forma sostenida, cada estudiante realiza la búsqueda bibliográfica que permite la clasificación y el ordenamiento de la información, contextualizando así, los objetos de enseñanza de manera que se constituyen en aprendizajes significativos. Se usan técnicas de observación y recogida de datos para la argumentación y formulación de una propuesta de modelo ecológico industrial, que se constituye como una experiencia que favorece la apropiación del objeto de estudio. Estos elementos posibilitan la elaboración del reporte de investigación, que permite la estructuración de ideas de acuerdo a la información recogida y en torno a la problemática a resolver, integrando los objetos de enseñanza como elementos indispensables para la argumentación y solución de la misma. La exposición pública de los trabajos de investigación formativa y la inclusión de técnicas cualitativas de análisis de información, permite elaborar juicios críticos sobre la validez del aprendizaje.

\section{RESULTADOS}

Para el logro de los objetivos propuestos, se desarrolla el diseño e intervención en aula de acuerdo al paradigma de la Sociedad del Conocimiento y siguiendo la estructura del Modelo T de programación propuestos por [11] y destacados por [12]. Así se tiene en la tabla 1 el diseño del modelo T para la asignatura en estudio.

Tabla 1. Diseño del Aprendizaje para la asignatura de Ecología Industrial (16 sesiones)

\begin{tabular}{|c|c|}
\hline CONTENIDOS & $\begin{array}{l}\text { ESTRATEGIAS TÉCNICAS I } \\
\text { EVALUACIÓN }\end{array}$ \\
\hline $\begin{array}{l}\text { 1) Cambio climático y efecto } \\
\text { invernadero. Orientación PNUMA } \\
\text { para el crecimiento industrial }\end{array}$ & $\begin{array}{l}\text { 1.- Separatas y documentos } \\
\text { en aula virtual / Registro } \\
\text { virtual de desempeño }\end{array}$ \\
\hline $\begin{array}{l}\text { 2) Teoría de Desarrollo sostenible. } \\
\text { Fundamentos de Ecología } \\
\text { Industrial. Modelos ecológicos } \\
\text { Industriales Huella Ecológica y } \\
\text { sostenibilidad. Capacidad de }\end{array}$ & $\begin{array}{l}\text { 2.- Lectura comprensiva: } \\
\text { esquema y extracción de } \\
\text { ideas principales. } \\
\text { Intervención Oral }\end{array}$ \\
\hline arga. Metabolismo Industrial & 3.- Análisis casos / Discusión \\
\hline
\end{tabular}

\begin{tabular}{|c|c|}
\hline Peruano. & y Reporte escrito en \\
\hline $\begin{array}{l}\text { 3) Simbiosis industrial. Casos: } \\
\text { Kalundborg y Kwinana } \\
\text { 4) Des-materialización. Des- } \\
\text { carbonización. Caso Minería } \\
\text { Peruana. - La Oroya } \\
\text { 5) Energías renovables y } \\
\text { alternativas. Combustibles } \\
\text { ecológicos. El proceso de des- } \\
\text { carbonización en el sistema } \\
\text { peruano. Perspectivas sostenibles } \\
\text { para la generación de energía. } \\
\text { Pilas de Hidrógeno. Pilas de } \\
\text { combustible: tecnología limpia } \\
\text { 6). Ciclo de vida de productos, } \\
\text { envases y embalaje } \\
\text { 7) Diseño para el medio ambiente. } \\
\text { Productos normados por el medio } \\
\text { ambiente }\end{array}$ & $\begin{array}{l}\text { 4.- Exposición final del } \\
\text { trabajo I Interrogación } \\
\text { elaborativa } \\
\text { 5.- Diseño del trabajo de } \\
\text { investigación formativa } \\
\text { referida la aplicación de } \\
\text { lineamientos de Ecología } \\
\text { Industrial en la evolución de } \\
\text { modelos ecológicos y a los } \\
\text { ciclos de vida de los } \\
\text { productos para una industria } \\
\text { en particular. / Reporte } \\
\text { escrito vía virtual de acuerdo } \\
\text { a las consideraciones } \\
\text { establecidas. } \\
\text { 7.- Presentación de videos y } \\
\text { software educativos / Informe } \\
\text { de desarrollo de actividad. } \\
\text { 8.- Visita técnica a una } \\
\text { industria regional / Informe } \\
\text { de visita técnica }\end{array}$ \\
\hline \multicolumn{2}{|c|}{ OBJETIVOS } \\
\hline CAPACIDADES & VAL \\
\hline $\begin{array}{l}\text { a)Recepción: } \\
\text {-Observación - Lectura } \\
\text {-Identificación - Interrogación } \\
\text { b)Reflexión: } \\
\text {-Análisis Comparación } \\
\text {-Formación de conceptos - } \\
\text { Síntesis } \\
\text { - Interpretación - Evaluación } \\
\text { crítica } \\
\text { - Argumentación } \\
\text { c)Creación: } \\
\text { - Originalidad } \\
\text { d)Retención: - Cambio } \\
\text { - Fijación - Evocación } \\
\text { e)Expresión práctica: } \\
\text {-Aplicación de conocimientos a } \\
\text { problemas concretos. } \\
\text { - Expresión oral y escrita }\end{array}$ & $\begin{array}{l}\text { a)Intelectuales: } \\
\text { - Hábitos de estudio } \\
\text { - Claridad de expresión } \\
\text { - Adhesión a la verdad } \\
\text { - Rigor científico } \\
\text { b)Prudenciales: } \\
\text { - Iniciativa - Audacia en } \\
\text { los proyectos } \\
\text { c)Sociales: } \\
\text {-Veracidad - Generosidad } \\
\text {-Trabajo en equipo } \\
\text { d)Individuales: } \\
\text { - Perseverancia } \\
\text { - Esfuerzo } \\
\text { - Dominio de sí mismo } \\
\text { - Sencillez } \\
\text {-Autonomía }\end{array}$ \\
\hline
\end{tabular}

En la elaboración de la monografía investigativa, la fuente de problemas es la que ofrecen los sectores industriales, así los trabajos de investigación están orientados al análisis de determinada situación problemática en una industria en particular, para proponer alternativas de desarrollo industrial denominados modelos ecológicos industriales como soporte para la toma de decisiones. Los temas a investigar son elegidos al inicio de la asignatura, de manera que son presentados y discutidos en aula según un cronograma de actividades establecido, así mismo mediante asesorías se orienta la ejecución de la investigación formativa.

En los instrumentos de evaluación empleados, los ítems confeccionados se orientan al análisis, la comparación, la síntesis y la interpretación de la información con que se cuenta. Así mismo, se brindan espacios de discusión en pares, se promueve el trabajo individual y autónomo así como la solidaridad y trabajo cooperativo. Es condición necesaria que el estudiante realice lectura previa de las separatas asignadas a fin de contribuir a la discusión en aula.

Así mismo, es necesario la formulación de logros e indicadores de aprendizaje que orientan la formulación de ítems en los instrumentos de evaluación formativa y 
continua, tal como se observa a continuación en la tabla 2.

Tabla 2. Logros de Aprendizaje - Instrumentos de Evaluación: Ecología Industrial

\begin{tabular}{|c|c|c|}
\hline $\begin{array}{ll}\text { Logros } & \text { de } \\
\text { aprendizaje } & \end{array}$ & Indicadores de logro & $\begin{array}{l}\text { Instrumentos } \\
\text { Evaluación } \\
\text { Formativa }\end{array}$ \\
\hline $\begin{array}{l}\text { LA1. Propone la } \\
\text { evolución de } \\
\text { modelos } \\
\text { ecológico } \\
\text { industriales, } \\
\text { tomando en } \\
\text { cuenta las } \\
\text { interacciones } \\
\text { con la biosfera, } \\
\text { bajo un criterio } \\
\text { de armonía con } \\
\text { la naturaleza }\end{array}$ & 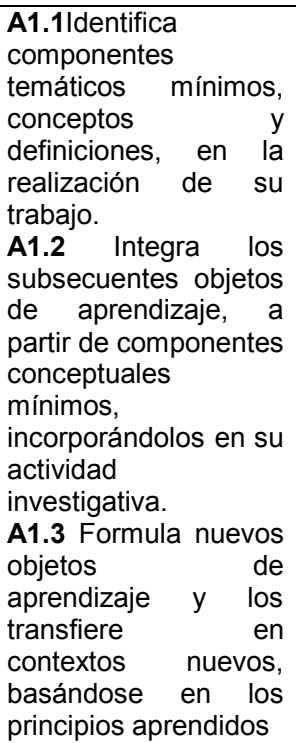 & $\begin{array}{l}\text { - Reporte de } \\
\text { investigación } \\
\text { presentado vía aula } \\
\text { virtual, de acuerdo a } \\
\text { las normas } \\
\text { establecidas } \\
\text { - Asistencia } \\
\text { asesoría } \\
\text { - Intervenciones } \\
\text { orales } \\
\text { - Pruebas de libro } \\
\text { abierto individuales y } \\
\text { en pares } \\
\text { - Exposiciones orales } \\
\text { - Revisión continua } \\
\text { de trabajos cortos } \\
\text { - Fichas de } \\
\text { evaluación }\end{array}$ \\
\hline $\begin{array}{l}\text { LA2. Desarrolla } \\
\text { conciencia } \\
\text { ambiental, } \\
\text { mediante el } \\
\text { análisis de los } \\
\text { objetos de } \\
\text { estudios de la } \\
\text { ecología } \\
\text { industrial, } \\
\text { incorporándolos } \\
\text { en el desarrollo } \\
\text { de su trabajo de } \\
\text { investigación. }\end{array}$ & $\begin{array}{l}\text { A2.1 Actúa con } \\
\text { autonomía r y } \\
\text { responsabilidad con el } \\
\text { medioambiente. } \\
\text { A2.2 Desarrolla } \\
\text { nuevas ideas y } \\
\text { enfoques sobre como } \\
\text { orientar su trabajo, } \\
\text { tomando en cuenta } \\
\text { los contenidos de } \\
\text { Ecología Industrial. } \\
\text { A2.3 Justifica la } \\
\text { evolución de los } \\
\text { modelos ecológicos } \\
\text { industriales, tomado } \\
\text { como base la } \\
\text { reutilización, reciclaje, } \\
\text { recuperación y las } \\
\text { emisiones cero. }\end{array}$ & $\begin{array}{l}\text {-Observación directa: } \\
\text { Escala de valoración } \\
\text {-Observación } \\
\text { indirecta: análisis del } \\
\text { trabajo }\end{array}$ \\
\hline
\end{tabular}

En el desarrollo de la asignatura de Ecología Industrial, los resultados finales se obtienen para cada estudiante y logro establecido, por indicador, por instrumento y por ítem. En este trabajo la monografía investigativa o reporte de investigación formativa, se evalúa de acuerdo a una estructura dada y asociada a los indicadores de logro.

La Tabla 3 relaciona los indicadores de logro de aprendizaje de contenidos, capacidades y valores en el marco de la elaboración de la monografía investigativa, de manera que dicha elaboración condense la totalidad de la asignatura en un trabajo de investigación específico. De manera que cada estudiante presenta un reporte de investigación formativa, los cuales una vez consolidados en un único documento por cada semestre son colocados en biblioteca virtual USAT (http://www.usat.edu.pe/campusvirtual/librerianet/investig aciones/investigaciones.aspx), para la lectura pública.

Los logros alcanzados en dichas elaboraciones, se determinan a partir de la información presentada por cada estudiante, instrumento e ítem, los cuales luego de ser registrados y analizados, se presentan consolidados en las Tabla 4 y Tabla 5 de forma resumida.

Tabla 3. Indicadores de Logro asociados a Monografía Investigativa

\begin{tabular}{|c|c|}
\hline Indicadores de logro & $\begin{array}{c}\text { Estructura del Reporte de } \\
\text { Investigación }\end{array}$ \\
\hline $\begin{array}{l}\text { A1.1 Identifica } \\
\text { componentes } \\
\text { temáticos mínimos, } \\
\text { conceptos la } \\
\text { definiciones, en la } \\
\text { realización de su } \\
\text { trabajo. } \\
\text { A1.2 Integra los } \\
\text { subsecuentes objetos } \\
\text { de aprendizaje, a } \\
\text { partir de componentes } \\
\text { conceptuales } \\
\text { mínimos, } \\
\text { incorporándolos en su } \\
\text { actividad investigativa. } \\
\text { A1.3 Formula nuevos } \\
\text { objetos de aprendizaje } \\
\text { y los transfiere en } \\
\text { contextos nuevos, } \\
\text { basándose en los } \\
\text { principios aprendidos }\end{array}$ & $\begin{array}{l}\text { Resumen: Contiene los objetivos y los } \\
\text { resultados de la investigación. } \\
\text { Palabras claves: 04 palabras que } \\
\text { representan el trabajo realizado } \\
\text { I.- Introducción: es la presentación del } \\
\text { tema a desarrollar y de los puntos de } \\
\text { vista desde los que se realiza el } \\
\text { análisis. } \\
\text { II.- Marco Teórico: Descripción general de } \\
\text { la Industria Manufacturera y de los } \\
\text { Productos } \\
\text { III.- Caracterización Físico química de } \\
\text { materia prima e insumos (señalar si } \\
\text { son tóxicos y/o contaminantes). } \\
\text { IV.- Caracterización Físico química de } \\
\text { emisiones, efluentes, residuos } \\
\text { industriales aprovechables y no } \\
\text { aprovechables (señalar si son tóxicos } \\
\text { y/o contaminantes). } \\
\text { V.- Argumentos de Ecología Industrial } \\
\text { que orientan la evolución hacia el } \\
\text { modelo ecológico Tipo II } \\
\text {-Diseño de Modelo Ecológico Tipo II } \\
\text { que orientan la evolución hacia el } \\
\text { Modelo Ecológico Tipo III }\end{array}$ \\
\hline $\begin{array}{l}\text { A2.1 Actúa con } \\
\text { autonomía } \\
\text { responsabilidad con el } \\
\text { medioambiente. }\end{array}$ & $\begin{array}{l}\text {-Diseño Modelo Ecológico Tipo III (Tipo } \\
\text { Eco parque, considerar cero } \\
\text { emisiones, uso de energías } \\
\text { renovables, reaprovechamiento, Re- } \\
\text { uso, Reciclaje, Recuperación, Des } \\
\text { materialización, etc.) }\end{array}$ \\
\hline $\begin{array}{l}\text { A2.2 Desarrolla nuevas } \\
\text { ideas y enfoques } \\
\text { sobre como orientar } \\
\text { su trabajo, tomando } \\
\text { en cuenta los } \\
\text { contenidos de } \\
\text { Ecología Industrial. }\end{array}$ & $\begin{array}{l}\text { VII.- Análisis del Ciclo de Vida de los } \\
\text { Productos (elaborar diagrama en } \\
\text { detalle, argumentar) } \\
\begin{array}{l}\text { Conclusiones (elaborar cuatro } \\
\text { conclusiones como mínimo que } \\
\text { respondan a los objetivos planteados) }\end{array}\end{array}$ \\
\hline $\begin{array}{l}\text { A2.3 Justifica la } \\
\text { evolución de los } \\
\text { modelos ecológicos } \\
\text { industriales, tomado } \\
\text { como base la } \\
\text { reutilización, reciclaje, } \\
\text { recuperación y las } \\
\text { emisiones cero }\end{array}$ & $\begin{array}{l}\text { Referencias Bibliográficas (se elaboran } \\
\text { estrictamente de acuerdo a norma } \\
\text { Chicago) }\end{array}$ \\
\hline
\end{tabular}

Tabla 4. Resumen de Resultados de logro en la Monografía Investigativa en porcentajes (25 estudiantes ciclo 2007-II)

\begin{tabular}{|l|l|l|l|l|l|l|}
\hline $\begin{array}{l}\text { Logros/ } \\
\text { Indicador } \\
\text { de Logro }\end{array}$ & A1.1 & $\begin{array}{l}\text { A1. } \\
2\end{array}$ & $\begin{array}{l}\text { A1. } \\
3\end{array}$ & $\begin{array}{l}\text { A2. } \\
1\end{array}$ & $\begin{array}{l}\text { A2. } \\
2\end{array}$ & $\begin{array}{l}\text { A2. } \\
3\end{array}$ \\
\hline LA1 & 90 & $\mathbf{8 5}$ & $\mathbf{8 5}$ & - & - & - \\
\hline LA2 & - & - & - & 90 & $\mathbf{8 0}$ & $\mathbf{8 0}$ \\
\hline
\end{tabular}


Tabla 5. Resumen de Resultados de logro en la Monografía Investigativa en porcentajes

(45 estudiantes ciclo 2008-I)

\begin{tabular}{|l|l|l|l|l|l|l|}
\hline $\begin{array}{l}\text { Logros/ } \\
\text { Indicador } \\
\text { de Logro }\end{array}$ & $\begin{array}{l}\text { A1. } \\
\mathbf{1}\end{array}$ & $\begin{array}{l}\text { A1. } \\
\mathbf{2}\end{array}$ & $\begin{array}{l}\text { A1. } \\
\mathbf{3}\end{array}$ & $\begin{array}{l}\text { A2. } \\
\mathbf{1}\end{array}$ & $\begin{array}{l}\text { A2. } \\
\mathbf{2}\end{array}$ & $\begin{array}{l}\text { A2. } \\
\mathbf{3}\end{array}$ \\
\hline LA1 & $\mathbf{9 0}$ & $\mathbf{9 0}$ & $\mathbf{8 6}$ & - & - & - \\
\hline LA2 & - & - & - & $\mathbf{9 3}$ & $\mathbf{8 6}$ & $\mathbf{8 5}$ \\
\hline
\end{tabular}

\section{DISCUSIÓN}

A partir de los resultados obtenidos mediante cada instrumento de evaluación y en particular en aquellos que evalúan los indicadores de logro para la monografía investigativa o trabajo de investigación formativa, se observa que los estudiantes están familiarizados con la sistematización y rigor que exige la investigación, así como con sus conceptos y metodología, y que han sido capaces de integrar progresivamente los contenidos propios de la asignatura en una aplicación industrial en particular.

Mediante los reportes de investigación elaborados y que hacen operativos los procesos de investigación formativa, se evidencia que ella posibilita la formación de profesionales de acuerdo a la visión de la USAT y que la sociedad necesita, al crear espacios que posibilitan integrar conocimientos con la aplicación específica de los mismos, de forma que el estudiante realiza acciones de práctica, que le permiten desarrollar competencias para la realización de investigación en sentido estricto. La generación de situaciones problemáticas de Ecología Industrial, en torno a una industria en particular, ha permitido al estudiante organizar y desarrollar conocimientos, aunque sean conocimientos ya existentes, aplicándolos en torno a sus expectativas y condiciones académicas profesionales específicas. De forma que coincidimos con [13], ya que la investigación formativa ha trascendido su función docente, convirtiéndose en una estrategia de mayor alcance: lograr una formación universitaria basada en el trabajo científico de los estudiantes.

Coincidimos con [14] cuando propone este tipo de investigación formativa como una herramienta motivacional relevante, pues mediante la intervención en aula durante los semestres 2007- II y 2008-I, se observa que la mayoría de los estudiantes logra realizar una búsqueda apropiada de aquellos elementos para la argumentación que les permite desarrollar propuestas innovadoras, a partir del interrogante inicial de trabajo. Así mismo en el reporte final elaborado que consolida tanto el marco teórico, la metodología de investigación y una aplicación industrial concreta, se observa que existe un buen nivel tanto interpretación y adquisición de conocimientos como en el desarrollo de la responsabilidad ambiental, observándose sin embargo dificultades en la redacción de las conclusiones.

Finalmente dada la diversidad de industria manufacturera, los reportes de investigación formativa elaborado son muy variados y apreciablemente buenos, por lo que es probable que florecerá mañana la investigación científica productiva tal como menciona [15].
Al intervenir en forma orientativa en aula, y empleando la asesoría y entrevista personal como estrategias se procura mejorar las actitudes de los estudiantes hacia la ciencia y el medio ambiente, integrando al estudiante en un proceso que requiere abordar la solución de un problema a través de la búsqueda de explicaciones siempre hipotéticas- que servirán para comprender el mundo real desde el punto de vista de la ingeniería. De esta forma coincidimos con [16] cuando afirman que sólo haciendo que los estudiantes realicen la práctica orientados por el profesor- de aspectos fundamentales de la metodología científica, se podrá superar su metodología de la superficialidad y, consecuentemente, construir conocimientos. Además se desarrolla un proceso de formación en investigación análogo a la formación inicial de futuros investigadores, en este aspecto es que se tienen coincidencias con el modelo de aprendizaje denominado investigación orientada.

\section{CONCLUSIONES}

- Mediante la monografía investigativa se hacen operativos los procesos de investigación formativa, posibilitándose la apropiación de saberes superiores, llámese contenidos de Ecología Industrial, mediante la aplicación práctica del mismo.

- La intervención en aula durante el desarrollo de la asignatura de Ecología Industrial del III ciclo de formación profesional en Ingeniería Industrial, se sustenta en el trabajo científico de investigación, logrando que los estudiantes participen en ella según sus expectativas personales.

- El desarrollo del binomio formación profesional e investigación formativa, con estudiantes de Ingeniería y en la asignatura de Ecología Industrial, permite tanto al profesor como al estudiante insertarse a la cultura de la investigación, al acentuar el carácter científico del conocimiento, promoviendo tanto el aprendizaje autónomo, como la curiosidad y rigor intelectual.

- La investigación formativa como eje articular de la enseñanza aprendizaje de las asignaturas Ingeniería, en particular de Ecología Industrial, posibilita el aprendizaje significativo en los estudiantes de Ingeniería Industrial. Así se destaca la investigación formativa como una estrategia pedagógica para el desarrollo del currículo.

- Esta estrategia pedagógica, posibilita en el profesor universitario el desarrollo de conocimientos prácticos educativos, integrando dichas acciones a su experiencia profesional, e incorporándolos a su ejercicio docente, de manera que se imparte una formación universitaria de carácter profesional sustentada en el trabajo científico, procurando así el desarrollo armónico, tanto de su labor investigativa como de su tarea docente.

- El proceso secuenciado de investigación formativa en la asignatura de Ecología Industrial, posibilita la mejora de la actitud responsable con el uso de los recursos naturales y energéticos, así como en el aprovechamiento y/o tratamiento de los residuos industriales, evidenciando en el diseño del reporte final, el uso de criterios de sostenibilidad. 


\section{REFERENCIAS BIBLIOGRÁFICAS}

[1] P. Vega Marcote, M. Freitas, P. Álvarez Suárez y R. Fleuri, R.. Marco Teórico y Metodológico de Educación Ambiental e Intercultural para un Desarrollo Sostenible. Revista Eureka Enseñanza y Divulgación Cien., 2007, 4(3), pp. 539-554

[2][6][15] B. Restrepo. Conceptos y aplicaciones de la investigación formativa, y criterios para evaluar la Investigación Científica en sentido estricto. Documento CNA Colombia: CORCAS Editores. 2003

[3] G. Bravo Acevedo. La UMCE, la investigación universitaria y la sociedad del conocimiento. IntraMuros, 2003, 3 (12), 60-64.

[4][7][8][13] C. Parra Moreno. Apuntes sobre la investigación formativa. Revista de Educación y Educadores, 2004, 4, $57-77$

[5][14] C. Furió , J. Guisasola J. La Enseñanza del Concepto de Campo Eléctrico Basada en un Modelo de Aprendizaje como Investigación Orientada. Enseñanza de las Ciencias, 2001, 19 (2). p. 319-334

[9] H. Tibbs. Industrial Ecology: An Environmental Agenda for Industry. Annals of Earth, 199311 (1), pp 3-28

[10]A. Vilches, D. Gil Pérez, J. C. Toscazo y O. Macías, Educación para la Sostenibilidad» [artículo en línea]. OEl. 1993 [Fecha de consulta: 31/10/08]. http://www.oei.es/decada/accion004.htm

[11] M. Román Pérez. Sociedad del Conocimiento y Refundación de la escuela desde el aula. Ediciones Amigo. 2004

[12] J. López Camps. I. Leal. Cómo aprender en la sociedad del conocimiento. Gestión 2000. 2002

[16]Vargas Hernández, J., Caycedo Lozano, L. 2006 Investigación formativa: algunas alternativas de acción con estudiantes de Bacteriología y Laboratorio Clínico. Nova Vol.4 Nro. 6 Junio - Diciembre de 2006:1-114

Email: ssalazar@usat.edu.pe 